\title{
FIXED POINTS OF ANTITONE MAPPINGS
}

\section{ZAHAVA SHMUELY}

ABSTRACT. We present a family of antitone mappings defined on complete atomic lattices which have the fixed point property. Two commuting mappings of the family have a common fixed point. An example is given of three commuting mappings of the family which do not have a common fixed point.

1. Tarski [4], Abian and Brown [1], and others have studied fixed points of isotone mappings on partially ordered sets. In [2] fixed points of certain antitone mappings are studied. Here we are dealing with a family of symmetric polarized mappings (see definitions below) which have the fixed point property. Basically, these mappings imitate the behaviour of the centralizer operator acting on the subsets of a given groupoid.

In this note $A$ denotes a complete lattice unless otherwise stated. An antitone mapping $T: A \rightarrow A$ is polarized if there exists an (necessarily unique) antitone mapping denoted by $T^{*}: A \rightarrow A$ such that $\left\langle T, T^{*}\right\rangle$ is a Galois connection on $A$, i.e., $T T^{*}(p) \geq p$ and $T^{*} T(p) \geq p$ for each $p \in A$. $L(A)$ is the set of polarized mappings $T: A \rightarrow A$. As is well known [3], $T$ $\in L(A)$ iff

(1) $T(0)=1$ where 0,1 are the universal bounds of $A$, and

(2) $T\left(\bigwedge_{p \in A^{\prime}} p\right)=\bigwedge_{p \in A^{\prime}}, T(p)$ for each $A^{\prime} \subseteq A$.

$T \in L(A)$ is symmetric if $T=T^{*}$. For $T \in L(A)$, let $p_{T}(A)$ denote the family of subsets $A_{i} \subseteq A$ satisfying $T\left(\bigvee_{p \in A_{i}} p\right) \geq \bigvee_{p \in A_{i}} p$. Notice that $\{0\} \in P_{T}(A) . P_{T}(A)$ is ordered by set inclusion. We now prove

Lemma 1. Let $A$ be a complete lattice and let $T \in L(A)$. Then $P_{T}(A)$ contains a maximal element.

Proof. Let $\left\{A_{j}\right\}_{j \in J}$ be a chain in $P_{T}(A)$, and put $\bigvee_{p \in A_{j}} p=p_{j}$. We now show that $A_{0}=\bigcup_{j \in J} A_{j}$ belongs to $P_{T}(A)$. Given any $i, j \in J$ we may assume $A_{i} \subseteq A_{j}$, and so $p_{i} \leq p_{j} \leq T\left(p_{j}\right) \leq T\left(p_{i}\right)$. It follows that $\bigvee_{i \in J} p_{i} \leq T\left(p_{j}\right)$ for each $j \in J$ and so $\bigvee_{i \in J} p_{i} \leq \bigwedge_{j \in J} T\left(p_{j}\right)$. By (2),

$$
\bigvee_{p \in A_{0}} p=\bigvee_{i \in J} p_{i} \leq T\left(\bigvee_{j \in J} p_{j}\right)=T\left(\bigvee_{p \in A_{0}} p\right)
$$

follows implying that $A_{0} \in P_{T}(A)$. By Zorn's lemma $P_{T}(A)$ contains a maximal element. 
Theorem 1. Let $A$ be a complete atomic lattice and let $T: A \rightarrow A$ be a symmetric polarized mapping satisfying $T(q) \geq q$ for each atom $q \in A$. Then $T$ has a fixed point.

Proof. Let $A_{0} \subseteq A$ be a maximal element of $P_{T}(A)$ (see Lemma 1), and put $p_{0}=\bigvee_{p \in A} p$. Obviously, $T\left(p_{0}\right) \geq p_{0}$. Assuming $T\left(p_{0}\right)>p_{0}$ we can find an atom $q \in A, q \notin A_{0}$ such that $T\left(p_{0}\right) \geq q$. Since $T=T^{*}, T(q) \geq p_{0}$ follows. This together with $T(q) \geq q$ yields $T\left(p_{0} \vee q\right)=T\left(p_{0}\right) \wedge T(q) \geq p_{0} \vee q$, contradicting the maximality of $A_{0}$. Thus $T\left(p_{0}\right)=p_{0}$ follows.

2. One is naturally led to consider commuting mappings having the properties specified in Theorem 1. Firstly, we have

Lemma 2. Let $A$ be a partially ordered set and let $T, S: A \rightarrow A$ be symmetric polarized mappings. If $T S=S T$ then $T^{2}=S^{2}$.

Proof. Since $S=S^{*}, T=T^{*}$ it follows that $T^{2}(p) \geq p, S^{2}(p) \geq p$ for each $p \in A$. Hence $T S T(p)=T^{2} S(p) \geq S(p)$. Since $S$ is antitone $T S T(p)=$ $S T^{2}(p) \leq S(p)$. Thus $T S T=S$ implying that $T^{2} S^{2}=S^{2}$. Similarly, we have $T^{2} S^{2}=\bar{T}^{2}$ and $T^{2}=S^{2}$ follows.

We can now prove

Theorem 2. Let $A$ be a complete atomic lattice and let $T, S: A \rightarrow A$ be symmetric polarized mappings satisfying $T(q) \geq q, S(q) \geq q$ for each atom $q \in A$. If $T S=S T$ then $T$ and $S$ possess a common fixed point.

Proof. The mapping $T \wedge S: A \rightarrow A$ defined by $(T \wedge S)(p)=T(p) \wedge S(p)$ is easily shown to satisfy $T \wedge S \in L(A)$ with $(T \wedge S)^{*}=T \wedge S$. Since $(T \wedge S)(q) \geq q$ for each atom $q \in A$, we may apply Theorem 1 to find $p_{0} \in A$ such that $(T \wedge S)\left(p_{0}\right)=p_{0}$. Since $T\left(p_{0}\right) \geq p_{0}, S\left(p_{0}\right) \geq p_{0}$ we get $S T\left(p_{0}\right) \leq$ $s\left(p_{0}\right), T S\left(p_{0}\right) \leq T\left(p_{0}\right)$ implying $s T\left(p_{0}\right) \leq S\left(p_{0}\right) \wedge T\left(p_{0}\right)=p_{0}$. Since $S=s^{*}$, $S=S^{3}$ follows. By Lemma $2, T^{2}=S^{2}$ holds. Thus, applying $T$ on the last inclusion we get

$$
S\left(p_{0}\right)=S^{3}\left(p_{0}\right)=T^{2} S\left(p_{0}\right)=T S T\left(p_{0}\right) \geq T\left(p_{0}\right) .
$$

$T\left(p_{0}\right) \geq S\left(p_{0}\right)$ follows similarly. Hence $T\left(p_{0}\right)=S\left(p_{0}\right)=(T \wedge S)\left(p_{0}\right)=p_{0}$, completing the proof.

The following example is brought to show that three commuting mappings, each satisfying the conditions of Theorem 1 , need not have a common fixed point.

Example. $A=2^{M}$ is the complete atomic Boolean lattice of subsets of $M=\{1,2,3,4,5,6\}$. For ease of notation let $m$ stand for the one-element set $\{m\}$ and let $p^{\prime}$ denote the complement of $p \in A$. The polarized mappings $R, S, T: A \rightarrow A$ are given by specifying their values on the atoms of $A$ (see 
(1), (2)) as follows:

$$
\begin{aligned}
& R: 1 \rightarrow 2^{\prime}, \quad 2 \rightarrow 1^{\prime}, \quad 3 \rightarrow 4^{\prime}, \quad 4 \rightarrow 3^{\prime}, \quad 5 \rightarrow 6^{\prime}, \quad 6 \rightarrow 5^{\prime} . \\
& S: 1 \rightarrow 2^{\prime}, \quad 2 \rightarrow 1^{\prime}, \quad 3 \rightarrow 5^{\prime}, \quad 4 \rightarrow 6^{\prime}, \quad 5 \rightarrow 3^{\prime}, 6 \rightarrow 4^{\prime} . \\
& T: 1 \rightarrow 2^{\prime}, \quad 2 \rightarrow 1^{\prime}, \quad 3 \rightarrow 6^{\prime}, \quad 4 \rightarrow 5^{\prime}, \quad 5 \rightarrow 4^{\prime}, 6 \rightarrow 3^{\prime} .
\end{aligned}
$$

Obviously, $R, S, T$ are symmetric and satisfy $R(q) \geq q, S(q) \geq q, T(q)$ $\geq q$ for each atom $q \in A$. By direct computation one can show that $R, S, T$ form a commutative family. The fixed points of $R$ are exactly those threeelement subsets of $M$ with each element belonging to a different cycle of the permutation which naturally corresponds to $R$ (namely (12) (34) (56)). A similar result holds for $S$ and $T$. An inspection of the corresponding cycles shows that $R, S, T$ do not possess a common fixed point.

Acknowledgement. The author wishes to thank Professor Abraham Ginzburg for his help in preparing this paper.

\section{REFERENCES}

1. S. Abian and A. B. Brown, A theorem on partially ordered sets, with applications to fixed point theorems, Canad. J. Math. 13 (1961), 78-82. MR 23 \#A817.

2. A. Gewirtz and L. V. Quintas, Antitone fixed point maps, Recent Trends in Graph Theory (Proc. Conf., New York, 1970), Lecture Notes in Math., vol. 186, Springer-Verlag, Berlin, 1971, pp. 117-136. MR $44 \# 121$.

3. G. Pickert, Bemerkungen über Galois॰Verbindungen, Arch. Math. 3 (1952), 285-289. MR 14, 529.

4. A. Tarski, A lattice-theoretical fixpoint theorem and its applications, Pacific J. Math. 5 (1955), 285-309. MR 17, 574.

DEP ARTMENT OF MATHEMATICS, TECHNION, ISR AEL INSTITUTE OF TECHNOLOGY, HAIF A, ISRAEL 